PROCEEDINGS OF THE

AMERICAN MATHEMATICAL SOCIETY

Volume 134, Number 5, Pages 1513-1517

S 0002-9939(05)08390-5

Article electronically published on October 7, 2005

\title{
A NOTE ON PERIODIC POINTS OF ORDER PRESERVING SUBHOMOGENEOUS MAPS
}

\author{
BAS LEMMENS AND COLIN SPARROW
}

(Communicated by Jonathan M. Borwein)

\begin{abstract}
Let $\mathbb{R}_{+}^{n}$ be the standard closed positive cone in $\mathbb{R}^{n}$ and let $\Gamma\left(\mathbb{R}_{+}^{n}\right)$ be the set of integers $p \geq 1$ for which there exists a continuous, order preserving, subhomogeneous map $f: \mathbb{R}_{+}^{n} \rightarrow \mathbb{R}_{+}^{n}$, which has a periodic point with period $p$. It has been shown by Akian, Gaubert, Lemmens, and Nussbaum that $\Gamma\left(\mathbb{R}_{+}^{n}\right)$ is contained in the set $B(n)$ consisting of those $p \geq 1$ for which there exist integers $q_{1}$ and $q_{2}$ such that $p=q_{1} q_{2}, 1 \leq q_{1} \leq\left(\begin{array}{c}n \\ m\end{array}\right)$, and $1 \leq q_{2} \leq\left(\begin{array}{c}m \\ \lfloor m / 2\rfloor\end{array}\right)$ for some $1 \leq m \leq n$. This note shows that $\Gamma\left(\mathbb{R}_{+}^{n}\right)=B(n)$ for all $n \geq 1$.
\end{abstract}

\section{INTRODUCTION}

Let $K$ be a polyhedral cone in a finite dimensional real vector space equipped with the norm topology and let $f: K \rightarrow K$ be a continuous map that preserves the ordering induced by $K$. Suppose, in addition, that $f: K \rightarrow K$ is subhomogeneous, that is, $\lambda f(x) \leq f(\lambda x)$ for all $0 \leq \lambda \leq 1$ and $x \in K$. It was proved by Akian et al. [2. Theorem 2.1] that every bounded orbit of $f: K \rightarrow K$ converges to a periodic orbit and, moreover, that the period of each periodic point of $f$ is bounded by

$$
\beta_{N}=\max _{1 \leq m \leq N}\left(\begin{array}{l}
N \\
m
\end{array}\right)\left(\begin{array}{c}
m \\
\lfloor m / 2\rfloor
\end{array}\right)=\frac{N !}{\left\lfloor\frac{N}{3}\right\rfloor !\left\lfloor\frac{N+1}{3}\right\rfloor !\left\lfloor\frac{N+2}{3}\right\rfloor !} \sim \frac{3^{N+1} \sqrt{3}}{2 \pi N},
$$

where $N$ is the number of facets of the polyhedral cone $K$. In fact, it follows from [2, Theorem 5.1] that the set of possible periods of periodic points of continuous, order preserving, subhomogeneous maps $f: K \rightarrow K$, denoted $\Gamma(K)$, is contained in a finite set $B(N)$ consisting of $p \geq 1$ for which there exist integers $q_{1}$ and $q_{2}$ such that $p=q_{1} q_{2}, 1 \leq q_{1} \leq\left(\begin{array}{c}N \\ m\end{array}\right)$, and $1 \leq q_{2} \leq\left(\begin{array}{c}m \\ \lfloor m / 2\rfloor\end{array}\right)$ for some $1 \leq m \leq N$ (compare [2, Section 7]). On the other hand, if the polyhedral cone $K$ is the standard positive cone given by $\mathbb{R}_{+}^{n}=\left\{x \in \mathbb{R}^{n}: x_{i} \geq 0\right.$ for $\left.1 \leq i \leq n\right\}$, then it follows from [2, Theorem 2.2] that $\Gamma\left(\mathbb{R}_{+}^{n}\right)$ contains the set $A(n)$ consisting of those $p \geq 1$ for which there exist integers $q_{1}$ and $q_{2}$ such that $p=\operatorname{lcm}\left(q_{1}, q_{2}\right)$, $1 \leq q_{1} \leq\left(\begin{array}{c}n \\ m\end{array}\right)$, and $1 \leq q_{2} \leq\left(\begin{array}{c}m \\ \lfloor m / 2\rfloor\end{array}\right)$ for some $1 \leq m \leq n$. As $\mathbb{R}_{+}^{n}$ has $n$ facets, we thus have the following inclusions:

$$
A(n) \subset \Gamma\left(\mathbb{R}_{+}^{n}\right) \subset B(n) \text { for all } n \geq 1 .
$$

Received by the editors November 23, 2004.

2000 Mathematics Subject Classification. Primary 54H20, 47H07; Secondary 15A48, 46T20.

Key words and phrases. Monotone dynamical systems, nonlinear Perron-Frobenius theory.

The first author was supported by a TALENT-Fellowship of the Netherlands Organization for Scientific Research (NWO). 
It has been shown in [2, Section 7] that the largest element of $A(n)$ has the same asymptotics as $\beta_{n}$ in (11), which is the largest element of $B(n)$. Knowing these facts it is natural to ask if one could completely determine the finite set $\Gamma\left(\mathbb{R}_{+}^{n}\right)$ and, in particular, to see if $\Gamma\left(\mathbb{R}_{+}^{n}\right)=A(n)$ or if $\Gamma\left(\mathbb{R}_{+}^{n}\right)=B(n)$. The purpose of this note is to show that $\Gamma\left(\mathbb{R}_{+}^{n}\right)$ and $B(n)$ are equal. The idea of the proof can be sketched as follows. First we take $q_{1}$, where $1 \leq q_{1} \leq\left(\begin{array}{c}n \\ m\end{array}\right)$, distinct parts of the cone, such that every element in these $q_{1}$ parts has exactly $m$ nonzero coordinates. Subsequently we select in each of the $q_{1}$ parts, $q_{2}$ distinct points, where $1 \leq q_{2} \leq\left(\begin{array}{c}m \\ \lfloor m / 2\rfloor\end{array}\right)$, and construct a continuous, order preserving, subhomogeneous map $f: \mathbb{R}_{+}^{n} \rightarrow \mathbb{R}_{+}^{n}$ which has the set of $q_{1} q_{2}$ points as a periodic orbit. It is known that $f$ has to map parts into parts and that the periodic orbit can have at most $\left(\begin{array}{c}m \\ \lfloor m / 2\rfloor\end{array}\right)$ points in a part of the cone that consists of elements with exactly $m$ nonzero coordinates (see [10, Theorem 5.2] and [2, Corollary 4.4]).

Before going into the details of the proof it is useful to recall several definitions. Let $X$ be a finite dimensional real topological vector space. A subset $K$ of $X$ is called a closed cone if it is a closed convex subset of $X$ such that $\lambda K \subset K$ for all $\lambda \geq 0$ and $K \cap(-K)=\{0\}$. A closed cone $K$ is called a polyhedral cone if there exist finitely many linear functionals $\varphi_{1}, \ldots, \varphi_{m}$ such that $K=\left\{x \in X: \varphi_{i}(x) \geq\right.$ 0 for $1 \leq i \leq m\}$. In other words, $K$ is the intersection of finitely many closed halfspaces. A face of a polyhedral cone is a set of the form $F=K \cap\{x \in X: \varphi(x)=0\}$, where $\varphi: X \rightarrow \mathbb{R}$ is a linear functional such that $K \subset\{x \in X: \varphi(x) \geq 0\}$. The dimension of a face is the dimension of its linear span and is denoted by $\operatorname{dim}(F)$. A face $F$ is called a facet if $\operatorname{dim}(F)=\operatorname{dim}(K)-1$.

A cone $K$ in a vector space $X$ induces a partial ordering $\leq$ on $X$ by $x \leq y$ if $y-x \in K$. A map $f: K \rightarrow K$ is called order preserving if for each $x, y \in K$ with $x \leq y$ we have that $f(x) \leq f(y)$. The map $f: K \rightarrow K$ is said to be subhomogeneous if $\lambda f(x) \leq f(\lambda x)$ for all $0 \leq \lambda \leq 1$ and $x \in K$. A point $x \in K$ is called a periodic point of $f: K \rightarrow K$ if $f^{p}(x)=x$ for some integer $p \geq 1$. The minimal such $p \geq 1$ is said to be the period of $x$ under $f$.

Maps that are order preserving and subhomogeneous arise in various areas of mathematics, including the theory of discrete event systems [3, 5, optimal control and game theory [1, idempotent analysis 8], nonlinear Perron-Frobenius theory [4, 12, and in the analysis of monotone dynamical systems [6, 7, 9, 11, 12, 13. A particularly interesting class of continuous, order preserving, homogeneous maps on the standard positive cone are the so-called min-max maps. To define this class of maps it is convenient to first introduce the following notation: for $a, b \in \mathbb{R}$ we write $a \wedge b$ to denote $\min \{a, b\}$ and $a \vee b$ to denote $\max \{a, b\}$. A min-max map $f: \mathbb{R}_{+}^{n} \rightarrow \mathbb{R}_{+}^{n}$ is a map such that each coordinate function $f_{i}(x)$ is of the form

$$
f_{i}(x)=\bigvee_{j=1}^{m}\left(\bigwedge_{k \in I_{j}} a_{j k}^{i} x_{k}\right),
$$

where each $I_{j}$ is a nonempty subset of $\{1, \ldots, n\}$ and every $a_{j k}^{i}>0$. Here the integer $m$ and the sets $I_{j}$ can depend on $i$. A simple example is the map $f: \mathbb{R}_{+}^{3} \rightarrow \mathbb{R}_{+}^{3}$ given by

$$
f\left(\begin{array}{l}
x_{1} \\
x_{2} \\
x_{3}
\end{array}\right)=\left(\begin{array}{c}
\left(2 x_{2} \wedge x_{1}\right) \vee\left(2 x_{1} \wedge x_{3}\right) \\
2 x_{3} \wedge x_{1} \\
2 x_{1} \wedge x_{2}
\end{array}\right) .
$$


It is easy to verify that $x=(1,2,0)$ is a periodic point of $f$ with period 4 . We shall use the min-max maps to prove the equality $\Gamma\left(\mathbb{R}_{+}^{n}\right)=B(n)$ by exhibiting for each $p \in B(n)$ a min-max map on $\mathbb{R}_{+}^{n}$ with a periodic point of period $p$.

\section{Proof of the Equality}

Having recalled these definitions we now prove the result of this note.

Theorem 2.1. For each $n \geq 1$ the sets $\Gamma\left(\mathbb{R}_{+}^{n}\right)$ and $B(n)$ are equal.

Proof. It has been shown in [2, Theorem 5.1] that $\Gamma\left(\mathbb{R}_{+}^{n}\right) \subset B(n)$ for all $n \geq 1$. To prove the opposite inclusion let $p \in B(n)$ and suppose that $p=q_{1} q_{2}$, where $q_{1}$ and $q_{2}$ are integers such that $1 \leq q_{1} \leq\left(\begin{array}{c}n \\ m\end{array}\right)$ and $1 \leq q_{2} \leq\left(\begin{array}{c}m \\ \mid m / 2\rfloor\end{array}\right)$ for some $1 \leq m \leq n$. We need to construct a continuous, order preserving, subhomogeneous map $f: \mathbb{R}_{+}^{n} \rightarrow \mathbb{R}_{+}^{n}$ and a periodic point of $f$ of period $p$.

We begin by constructing a periodic orbit of size $q_{2}$ in $\{1,2\}^{m}$. Let $w^{1}, \ldots, w^{q_{2}}$ be vectors in $\{1,2\}^{m}$ such that each $w^{k}$ has exactly $\lfloor m / 2\rfloor$ coordinates equal to 2 and put $w^{q_{2}+1}=w^{1}$. Note that there are $\left(\begin{array}{c}m \\ \lfloor m / 2\rfloor\end{array}\right)$ such elements in $\{1,2\}^{m}$ and hence we can do this for all possible values of $q_{2}$. It is easy to verify that $\left\{w^{1}, \ldots, w^{q_{2}}\right\}$ is a periodic orbit of the min-max map $g: \mathbb{R}_{+}^{m} \rightarrow \mathbb{R}_{+}^{m}$ given by

$$
g(z)_{i}=\bigvee_{k: w_{i}^{k+1}=2}\left(\bigwedge_{j: w_{j}^{k}=2} z_{j}\right), \quad 1 \leq i \leq m \text { and } z \in \mathbb{R}_{+}^{m} .
$$

Indeed, one can check that $g\left(w^{k}\right)=w^{k+1}$ for all $1 \leq k \leq q_{2}$.

The idea is to copy the points $w^{1}, \ldots, w^{q_{2}}$ into $q_{1}$ distinct parts of the cone $\mathbb{R}_{+}^{n}$ and subsequently to define a min-max map $f: \mathbb{R}_{+}^{n} \rightarrow \mathbb{R}_{+}^{n}$ that cycles through the $q_{1} q_{2}$ points. To do this we first select $q_{1}$ distinct subsets $I_{a}$ of $\{1, \ldots, n\}$ of size $m$. Each set $I_{a}$ corresponds to a part $P_{a}$ of the cone $\mathbb{R}_{+}^{n}$ by defining

$$
P_{a}=\left\{x \in \mathbb{R}_{+}^{n}: x_{i} \neq 0 \text { if and only if } i \in I_{a}\right\} .
$$

Now we copy the set $\left\{w^{1}, \ldots, w^{q_{2}}\right\}$ into each of the $q_{1}$ parts $P_{a}$ by inserting zero coordinates in the correct places. To do this formally it is convenient to introduce the following function. For $1 \leq a \leq q_{1}$ and $1 \leq k \leq m$ let $\nu(a, k)$ be the index of the $k$ th nonzero coordinate of the elements of $P_{a}$. So for each $1 \leq a \leq q_{1}$ we define the copying function $\eta_{a}: \mathbb{R}_{+}^{m} \rightarrow \mathbb{R}_{+}^{n}$ by

$$
\eta_{a}(z)_{i}= \begin{cases}z_{k} & \text { if } \nu(a, k)=i \\ 0 & \text { otherwise }\end{cases}
$$

By applying the functions $\eta_{a}$ to the set $\left\{w^{1}, \ldots, w^{q_{2}}\right\}$ we obtain $p=q_{1} q_{2}$ distinct points in $\mathbb{R}_{+}^{n}$. For $1 \leq a \leq q_{1}$ and $1 \leq b \leq q_{2}$ we let $y^{a, b} \in\{0,1,2\}^{n}$ denote the vector given by $y^{a, b}=\eta_{a}\left(w^{b}\right)$. Thus, $y^{a, b}$ is the copy of $w^{b}$ in the part $P_{a}$.

To complete the proof we define a min-max map $f: \mathbb{R}_{+}^{n} \rightarrow \mathbb{R}_{+}^{n}$ such that

$$
f\left(y^{a, b}\right)= \begin{cases}y^{a+1, b} & \text { if } 1 \leq a<q_{1}, \\ y^{1, b+1} & \text { if } a=q_{1},\end{cases}
$$

for each $1 \leq a \leq q_{1}$ and $1 \leq b \leq q_{2}$. (Here the index $b$ is counted modulo $q_{2}$.) It is clear that if $f$ satisfies (2), then $y^{a, b}$ is a periodic point of $f$ with period $p=q_{1} q_{2}$. 
Before we define the min-max map $f: \mathbb{R}_{+}^{n} \rightarrow \mathbb{R}_{+}^{n}$ we introduce the following notation. For each $x \in \mathbb{R}_{+}^{n}$ we let $x_{\mid I_{a}}$ denote the vector in $\mathbb{R}_{+}^{m}$ with coordinates $\left(x_{\mid I_{a}}\right)_{j}=x_{\nu(a, j)}$ for $1 \leq j \leq m$. Now let $f: \mathbb{R}_{+}^{n} \rightarrow \mathbb{R}_{+}^{n}$ be given by

$$
f(x)_{i}=\left[\bigvee_{1 \leq k<q_{1}}\left(\bigwedge_{j \in I_{k}} 2 x_{j}\right) \wedge \eta_{k+1}\left(x_{\mid I_{k}}\right)_{i}\right] \vee\left[\left(\bigwedge_{j \in I_{q_{1}}} 2 x_{j}\right) \wedge \eta_{1}\left(g\left(x_{\mid I_{q_{1}}}\right)\right)_{i}\right]
$$

for $1 \leq i \leq n$ and $x \in \mathbb{R}_{+}^{n}$. As $f$ is a min-max map, it is continous, order preserving, and homogeneous. To see that (2) holds for $f$, we remark that $\bigwedge_{j \in I_{k}} 2 y_{j}^{a, b}=0$ whenever $k \neq a$ and $\bigwedge_{j \in I_{k}} 2 y_{j}^{a, b}=2$ otherwise. We also have that $y_{\mid I_{a}}^{a, b}=w^{b}$ and therefore

$$
f\left(y^{a, b}\right)_{i}=\left(\bigwedge_{j \in I_{a}} 2 y_{j}^{a, b}\right) \wedge \eta_{a+1}\left(w^{b}\right)_{i}=2 \wedge y_{i}^{a+1, b}=y_{i}^{a+1, b}
$$

if $1 \leq a<q_{1}$. On the other hand, if $a=q^{1}$, then

$$
f\left(y^{a, b}\right)_{i}=\left(\bigwedge_{j \in I_{q_{1}}} 2 y_{j}^{q_{1}, b}\right) \wedge \eta_{1}\left(g\left(w^{b}\right)\right)_{i}=2 \wedge \eta_{1}\left(w^{b+1}\right)_{i}=y_{i}^{1, b+1}
$$

and this completes the proof.

We conclude by noting that there are other ways to cycle through the points $y^{a, b}$ by using different min-max functions. In fact the only restriction on the periodic orbit is that if two of its points are in the same part of the cone, then their images should also be contained in a single part of the cone.

\section{REFERENCES}

[1] M. Akian and S. Gaubert, Spectral theorem for convex monotone homogeneous maps, and ergodic control. Nonlinear Anal. 52(2), (2003), 637-679. MR1938367 (2003i:93085)

[2] M. Akian, S. Gaubert, B. Lemmens, and R. D. Nussbaum, Iteration of order preserving subhomogeneous maps on a cone, Math. Proc. Cambridge Philos. Soc., to appear.

[3] F. Baccelli, G. Cohen, G. J. Olsder, and J. P. Quadrat, Synchronization and Linearity: An Algebra for Discrete Event Systems, Wiley Ser. Probab. Statist. Probab. Statist., Wiley: Chichester, 1992. MR1204266 (94b:93001)

[4] S. Gaubert and J. Gunawardena, The Perron-Frobenius theory for homogeneous, monotone functions. Trans. Amer. Math. Soc., 356(12), (2004), 4931-4950. MR2084406

[5] J. Gunawardena, From max-plus algebra to nonexpansive mappings: a nonlinear theory for discrete event systems. Theoret. Comput. Sci. 293(1), (2003), 141-167. MR1957616 (2004b:93083)

[6] M. W. Hirsch, Positive equilibria and convergence in subhomogeneous monotone dynamics, in Comparison Methods and Stability Theory (X. Liu and D. Siegel ed.), pp. 169-188, Lecture Notes in Pure and Appl. Math., 162, Dekker, New York, 1994. MR.1291618 (95f:34090)

[7] J. F. Jiang, Sublinear discrete-time order-preserving dynamical systems. Math. Proc. Cambridge Philos. Soc. 119(3), (1996), 561-574. MR1357065 (96h:34090)

[8] V. N. Kolokoltsov and V. P. Maslov, Idempotent Analysis and Applications. Kluwer Acad. Press, 1997. MR 1447629

[9] U. Krause and P. Ranft. A limit set trichotomy for monotone nonlinear dynamical systems. Nonlinear Anal. 19(4), (1992), 375-392. MR1178411 (93i:58081)

[10] B. Lemmens and M. Scheutzow, On the dynamics of sup-norm nonexpansive maps, Ergodic Theory Dynam. Systems 25(3), (2005), 861-871. MR2142949

[11] R. D. Nussbaum, Hilbert's projective metric and iterated nonlinear maps. Mem. Amer. Math. Soc. 75 (391), (1988), 1-137. MR0961211 (89m:47046) 
[12] R. D. Nussbaum, Iterated nonlinear maps and Hilbert's projective metric II. Mem. Amer. Math. Soc. 79(401), (1989), 1-118. MR0963567 (90c:47109)

[13] P. Takáč, Asymptotic behavior of discrete-time semigroups of sublinear, strongly increasing mappings with applications to biology. Nonlinear Anal. 14(1), (1990), 35-42. MR1028245 (90j:47088)

Mathematics Institute, University of Warwick, Coventry, CV4 7AL, United Kingdom

E-mail address: lemmens@maths.warwick.ac.uk

Mathematics Institute, University of Warwick, Coventry, CV4 7AL, United Kingdom

E-mail address: csparrow@maths.warwick.ac.uk 